\title{
N76-10176
}

\section{COMPARISON THEOREMS, NUMERICAL INTEGRATION, AND SATELLITE ORBITS}

\author{
Arnold Stokes \\ Georgetown University \\ Washington, D.C.
}

Consider the equation

$$
\dot{\mathbf{x}}=\mathbf{X}(\mathbf{x}) \text {. }
$$

Assume that the behavior of the family of solutions is known, expressed by the estimate

$$
\left|x\left(t, x_{0}\right)-x\left(t, x_{1}\right)\right| \leqslant K_{0} f(t)\left|x_{1}-x_{0}\right|, t \geqslant 0,
$$

Here $K_{0}$ is constant, and $f$ is normalized by $f(0)=1, f(t) \geqslant 1$. The function $f(t)$ then expresses the stability or instability of ( 1 ), so that stability is equivalent to $f \equiv 1$, and a typical instability would be $f(t)=t+1$.

Suppose we wish to numerically compute a particular solution of $(1)$, say $x\left(t, x_{0}\right)$, for some fixed $x_{0}$. A remarkable theorem of Babuska asserts (under reasonable assumptions on $X$ ) that if a strongly stable difference scheme is used, numerical integration of (1) with $x(0)=x_{0}$ is equivalent to obtaining an exact (theoretical) solution of

$$
\dot{y}=X(y)+G(t, y),
$$

where $y(0)=x_{1}$, (here $\left|x_{1}-x_{0}\right|$ is the starting error), and $|G(t, y)| \leqslant \eta$, (here $\eta$ (small) reflects the discretization and truncation error).

Babuska used this result to obtain global error bounds for (2), assuming $x\left(t, x_{0}\right)$ is a uniformly asymptotically stable solurion of (1). Here we wish to compare solutions of (1) with solutions of (2), with particular attention given to the effect of different types of $f(t)$.

To develop the comparisoli, a converse theorem using Lyapunov functions due to Yoshizawa and Hale is applied. Let $z=x-x\left(t, x_{0}\right)$, then ( 1 ) becomes

$$
\dot{z}=\mathbf{Z}(\mathrm{t}, \mathrm{z}) \text {, }
$$

and the new estimate becornes

$$
\left|z\left(t, t_{0}, z_{0}\right)-z\left(t, t_{0}, z_{1}\right)\right|<K_{0} f(t)\left|z_{1}-z_{0}\right|
$$




$$
\begin{aligned}
& =K_{0} f\left(t_{0}\right) \frac{f(t)}{f\left(t_{0}\right)}\left|z_{1}-z_{0}\right| \\
& =K\left(t_{0}\right)\left|z_{1}-z_{0}\right| \exp \left(\alpha(t)-\alpha\left(t_{0}\right)\right)
\end{aligned}
$$

where $K\left(t_{0}\right)=K_{0} f\left(t_{0}\right), \alpha(t)=\log f(t)$.

Then one can show there exists a function $V(t, z)$ satisfying

$$
\begin{aligned}
|z| & \leqslant V(t, z) \leqslant K(t)|z| \\
|V(t, z)-V(t, y)| & \leqslant K(t) \quad|z-y|, \text { and } \\
\dot{V}_{3} \mid & \leqslant \dot{\alpha} V=\frac{\dot{f}}{f} V
\end{aligned}
$$

where $\dot{V}_{3}$ denotes the derivative of $V$ along solutions of (3). Note that (a) and (c) allow one to recover (4), using standard theorems. Further, it is easy to show, using (b) that

$$
\dot{\mathrm{V}}_{2} \leqslant \dot{\alpha} V+K(t) \eta=\frac{\dot{\mathrm{f} V}}{\mathrm{t}}+\mathrm{K}_{0} \mathrm{f}(\mathrm{t}) \eta
$$

Then an immediate consequence is that

$$
\left|y\left(t, x_{1}\right)-x\left(t, x_{0}\right)\right|<x_{0} f(t)\left[\left|x_{1}-x_{0}\right|+\eta t\right]
$$

So we see that the term $K_{0} f(t)\left|x_{1}-x_{0}\right|$ reflects the propagation of the starting error, and the term $\eta \mathrm{K}_{0} \mathrm{f}(\mathrm{t})$ gives an estimate of the effects of the discretization and truncation error.

Evidently, it is better to integrate a stable system, $f \equiv 1$, than an unstable system, $f(t) \equiv t+1$. A simple example is the unperturbed two-body problem, which is unstable, as the difference between two periodic solutions of different periods grows (at least locally) like $t+1$. An example of a stable problem is the stabilized Kepler problem, as given hy Baumgarte and Stiefel, where now all solutions have the same period.

To extend the above to perturbed problems, consider

$$
\dot{x}=X(x)+\epsilon X_{1}(t, x)
$$

Assume that in some region $\mathrm{R},\left|\mathrm{X}_{1}(t, x)\right|=-\mathrm{M}$. Here one supposes this region $\mathrm{R}$ contdins the solution of interest and is large enough so that continued integration would not be of interest long before the integrated solution leaves $R$. 
This assumption will allow the effect of $f(t)$ on the error to be observed, for now we consider

$$
\dot{y}=X(y)+\epsilon X_{1}(t, y)+G(t, y)
$$

and $y(0)=x_{1},|G(t, y)| \leqslant \eta$ as before.

The same V-function leads to the estimate

$$
\left|y\left(t, x_{1}\right)-x\left(t, x_{0}\right)\right| \leqslant K_{0} f(t)\left[\left|x_{1}-x_{0}\right|+t(\eta+\epsilon M)\right],
$$

which is valid as long as both solutions remain in R. Ar,ain one can see the different error estimates obtained for $\mathrm{f} \equiv 1$ or $\mathrm{f}=\mathrm{t}+1$. Remark: The foregoing gives upper bounds on the error. The strength of the conclusion then rests on the sharpness of the upper bounds, for one cannot cciclude Error ${ }_{1}<$ Error $_{2}$ on the basis that a crude upper bound of the first is less than a crude upper bound of the second.

However, by considering simple examples, one can see that at least in these cases, numerical integration of a stable system gives an errcr growth $0(\eta t)$, while for an unstat ie prohlem, the error grows like $0\left(\eta t^{2}\right)$. So to that extent the foregoing estimates seem reasonable.

Further, numerical experiments are being conducted at Goddard Space Fiight Center using the stabilization techniques of Baumgarte and Stiefel, and the results should give further insight into the significance of the above results.

In this regard, perhaps it should be observed that a similar approach to obtain lower bounds does not seem possible, as the difference of two almost periodic functions will, in general, have an arbitrarily small lower bound.

Note that the above : imates can also be obtained in more complicated problems, with such means as time-dependent potentials. Details will appear in a forthcoming paper. 


\title{
OPTIMAL EXPLICIT RUNGE-KUTTA METHODS
}

\author{
D. Bettis and D. Hall \\ Unirersity of Texus \\ Austin. Texas
}

Optimal explicit Runge-Kutta methods are developed for soiving the initial value problem for systems of ordinary differential equations. These methods have an optimal estimate of the local truncation error term. thereby allowing the option of implementing a variablestep strategy. The coefficients of the Runge-Kutta method are selected so that the local truncation error is minimized and that the absolute stability region is maximized. 